\title{
Platón en Alemania Reflexiones en torno a la recepción de la doctrina platónica de las ideas en Kant y Wieland
}

\author{
Plato in Germany \\ Some remarks on the reception \\ of the platonic doctrine of ideas \\ in Kant and Wieland
}

\author{
MIQUEL SOLANS BLASCO \\ Universidad de Navarra
}

Recibido: 15/12/2017 Aceptado: 19/12/2017

\section{RESUMEN:}

En el presente artículo expongo, en primer lugar, la crítica por parte de Kant de las ideas platónicas, con el fin de mostrar el presupuesto interpretativo tradicional que concibe las ideas en Platón como correlatos objetivos de un acto de intuición. A continuación, expongo de modo general los límites hermenéuticos de dicha interpretación, en la medida en que no hace justicia a las formas de conocimiento no objetivistas que Platón introduce como el lugar en el que se conocen originariamente las ideas. Finalmente, desarrollo en sus puntos fundamentales la lectura no-objetivista de las ideas y de su conocimiento por parte de Wieland.

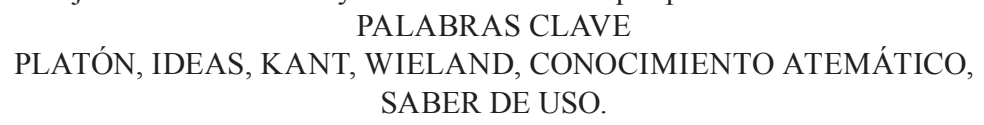

PALABRAS CLAVE

PLATÓN, IDEAS, KANT, WIELAND, CONOCIMIENTO ATEMÁTICO, SABER DE USO. 
ABSTRACT:

In this article I unfold, first, the Kantian critic of platonic ideas in order to show the traditional interpretative presupposition according to which ideas in Plato are to be understood as an objective correlate of an intuition. Next, I develop in general terms the hermeneutical limits of such interpretation, showing that this does not make justice to the non-objectivist forms of knowledge introduced by Plato as the locus in which ideas are originary understood. Finally, I present the fundamentals of Wieland's non-objectivistic interpretation of platonic ideas and their knowledge.

\section{KEYWORDS \\ PLATO, IDEAS, KANT, ATHEMATIC KNOWLEDGE, USE-KNOWLEDGE}

En el Reciente estudio de Alan Kim Plato in Germany (Kim 2010), el autor pone de relieve la deuda que guarda la interpretación de la «teoría de las ideas» de Platón en la filosofía alemana a partir del siglo XVIII con la recepción y el tratamiento de esta doctrina en la filosofía de Kant. ${ }^{1}$ La crítica kantiana -con claras resonancias aristotélicas ${ }^{2}$ - de este aspecto central de la filosofía platónica resulta tan caritativa en el tono ${ }^{3}$ como demoledora en su diagnóstico: ${ }^{4}$ según el pensador alemán, Platón habría confundido lo subjetivo -las ideas como principios regulativos del entendimiento- con lo objetivo -al atribuirles a éstas de forma ilegítima un estatuto sustancial-. Se trata del error que en la Dissertatio (1770) recibe el nombre de «ilusión metafísica» (24/II 412). La crítica kantiana se presenta como una rectificación de esta confusión central del platonismo, en la medida en que contribuye a poner de manifiesto los fundamentos subjetivos de aquello que en un acceso pre-crítico al mundo es tomado como algo objetivo. ${ }^{5}$

En la presente contribución, trato de mostrar, por un lado, que el presupuesto interpretativo fundamental de esta lectura de Platón, a saber, que las ideas son correlatos objetivos de intuiciones intelectuales, no es la única ni la que más justicia hace al papel que el filósofo griego atribuye a las formas en su filosofía y, por otro, que paradójicamente ha sido la reflexión kantiana acerca de las ideas como principios regulativos y de la facultad de juzgar la que ha contribuido de forma remota pero decisiva a esclarecer la genuina naturaleza de lo ideal en el pensamiento platónico.

Para ello, procederé en el siguiente orden: primero (1) expondré con algo más de detalle la naturaleza del error que según el filósofo alemán condujo a Platón a considerar las ideas como objetos dados a la intuición; a continuación,

1 Kim 2010, p. 22 y 45.

2 Cfr. Wieland 1999, p. 98.

3 Cfr. $K r V$ A318/B375.

4 Cfr. $K r V$ B9, A473-4/B499-500.

5 Cfr. KrV A296-7/B352-3; Kim 2010, p. 45. 
(2) haré referencia a los límites hermenéuticos de esta interpretación objetivista de las ideas platónicas; y, finalmente (3) mostraré, a partir de la interpretación de las ideas que ha propuesto Wolfgang Wieland, que cabe una lectura no-objetivista de lo ideal en Platón hermenéuticamente más fructífera que su tradicional asmiliación -compartida por Kant- a objetos dados a la intuición.

\section{LA LECTURA KANTIANA DE LAS IDEAS PLATÓNICAS EN LA CRÍTICA DE LA RAZÓN PURA.}

Según apunta Kant en la Crítica de la razón pura (en adelante $\mathrm{KrV}$ ), Platón usa el término idea (Idee) para designar un objeto que reúne las siguientes características:

1. No es tomado nunca de los sentidos. ${ }^{6}$

2. Sobrepasa los conceptos del entendimiento (die Begriffe des Verstandes) porque nunca se encuentra en la experiencia algo congruente con ello. ${ }^{7}$

3. Es un arquetipo de la cosa misma (Urbilde der Dinge selbst), y no solamente una clave de experiencias posibles (las ideas no son categorías). ${ }^{8}$

4. Emana de la razón suprema y de allí fue participada por la razón humana, participación que el hombre puede evocar y reactualizar en la medida en que filosofa. ${ }^{9}$ En este sentido, afirma también que se trata de «una idea del entendimiento divino, un objeto singular (ein einzelner Gegenstand) en la intuición pura de él, lo más perfecto de cada especie de entes posibles, y el fundamento originario (der Urgrund) de todas las copias en el fenómeno»..$^{10}$

5. Está dotada de fuerza creativa (schöpferische Kraft). ${ }^{11}$

6. La idea es un objeto (Gegenstand) del entendimiento puro (reine Verstand) que este presenta, sin mediación de los sentidos, por medio de una intuición (Anschauung). ${ }^{12}$

Según el filósofo alemán, la idea es la respuesta platónica a la necesidad intrínseca que impulsa a la mente humana hacia conceptos tan elevados que nunca podrán ser hallados como objetos de la experiencia, pero que sin embargo no dejan por ello de poseer cierta realidad (Realität) y que, en definitiva, no pueden tratarse de meras quimeras (bloße Hingerspinste). ${ }^{13}$ En su afán por salvar la validez de los contenidos con los que la razón se ve obligada a

6 Cfr. $K r V$ A313/B370.

7 Cfr. ibid..

8 Cfr. ibid..

9 Cfr. ibid..

$10 \mathrm{KrV}$ A568/B596. Sigo la traducción española de Caimi 2009.

11 Cfr. Idem A569/B597.

12 Cfr. Idem A853-4/B881-2.

13 Idem A314/B371. Cfr. A298/B354-5. 
operar tanto en su uso especulativo como práctico, ${ }^{14}$ Platón habría caído en la exageración de hipostasiar las ideas (...den Übertreibungen, dadurch er sie gleichsam hypostasirte). ${ }^{15}$

En efecto, a juicio de Kant, Platón habría transitado de forma inadvertida desde el pensamiento (denken) de la idea como principio regulativo del uso sistemático del entendimiento, es decir, como principio de unidad según el que pensar la multiplicidad del fenómeno, a su suposición como substancia simple (als einfache Substanz...ausnehmen). ${ }^{16}$

La idea es, según Kant, un concepto de la razón, es decir, un concepto que sobrepasa la posibilidad de la experiencia y que por ello mismo nunca puede ser dado en ella como objeto. Puesto que no hay intuición posible de la idea, la validez de un concepto tal no se debe por tanto a la existencia de un correlato objetivo de él, sino que su fundamento ha de ser necesariamente subjetivo, esto es, ha de encontrarse necesariamente en la estructura de la razón misma en cuanto facultad de conocer. ${ }^{17}$

En este sentido, Kant atribuye a Platón el comprensible pero grave error de haber caído víctima de la «apariencia ilusoria trascendental» (der trasscendentale Schein), cuya naturaleza explica como sigue:

...en nuestra razón (considerada subjetivamente como una facultad cognoscitiva humana [subjektiv als ein Erkenntnisvermögen betrachtet]) residen reglas fundamentales y máximas del uso de ella [las ideas], que tienen enteramente el aspecto de principios objetivos (das Ansehen objektiver Grundsätze haben), y por los cuales acontece que la necesidad subjetiva (die subjektive Notwendigkeit) de una cierta conexión de nuestros conceptos en beneficio del entendimiento, sea tenida por una necesidad objetiva (für eine objektive Notwendigkeit) de la determinación de las cosas en sí mismas. Una ilusión (Ilusion) que no se puede evitar, tal como no podemos evitar que el mar, hacia adentro, nos parezca más alto que en la costa, porque allá lo vemos mediante rayos luminosos más altos que a ésta; o aún más: tal como tampoco el astrónomo mismo puede impedir que la luna, al salir, le parezca más grande, aunque a él no le engañe esta apariencia ilusoria. ${ }^{18}$

14 Cfr. ibid. y A315/B372.

15 Idem, anotación al pie en A314/B371.

16 Cfr. idem A771/B799.

17 «[Los conceptos de la razón pura, i.e. las ideas,] no son fingidos arbitrariamente (willkürlich erdichtet), sino establecidos por la naturaleza de la razón misma (durch die Natur der Vernunft selbst aufgegeben), y se refieren necesariamente, por eso, a la totalidad del uso del entendimiento» (KrV A327/B384). Cfr. A321ss./B377ss.

$18 K r V$ A297/B353-4. 
Es decir, el error consiste en tomar la necesidad subjetiva conforme a la cual operan los enlaces de nuestra facultad de conocimiento por una necesidad objetiva en el orden de la constitución de las cosas mismas. Esta confusión viene motivada por la apariencia de objetividad que, por su propia condición como reglas del entendimiento -y por tanto del conocimiento objetivo- es connatural a las ideas de la razón. Únicamente el filósofo trascendental, en la medida en que advierte que las ideas valen no objetivamente como principio de la constitución de las cosas sino subjetivamente como reglas de su aparecer ante la conciencia objetiva, está en condiciones de tomar la inevitable apariencia objetiva con la que comparecen las ideas como mera apariencia, esto es, en su verdadero estatuto en cuanto determinación subjetiva del conocimiento objetivo.

En otras palabras, la naturaleza regulativa de las ideas kantianas explica por qué estas no pueden comparecer como un objeto de la experiencia y, al mismo tiempo, se prestan sin embargo de forma natural a ser tomadas precisamente como tales. ${ }^{19} \mathrm{Al}$ ser reglas del pensamiento objetivo, comparecen necesariamente proyectadas en él de modo que precisamente al regular lo objetivo, su propio carácter proyectivo queda oculto para una mirada que no esté avisada de la naturaleza originariamente subjetiva de los elementos que constituyen el campo de experiencia que se abre ante ella, es decir, para una consideración no trascendental o ingenua. Según la lectura kantiana, la doctrina de las ideas de Platón respondería de forma paradigmática a este último tipo de pensamiento, que como es sabido Kant califica también de pre-crítico o dogmático.

\section{LOS LÍMITES HERMENÉUTICOS DE LA INTERPRETACIÓN TRADICIONAL DE LAS IDEAS PLATÓNICAS.}

En uno de los pasajes de la $\mathrm{KrV}$ en los que Kant trata con más detalle de la teoría de las ideas de Platón, el filósofo alemán introduce la observación de que resulta perfectamente normal llegar a «entender a un autor, mediante la comparación de los pensamientos que expresa sobre su objeto, mejor aún de lo que él se entendió a sí mismo, si es que no determinó suficiente su concepto, y por ello, a veces habló, o aun pensó, en contra de su propia intención». ${ }^{20}$ Es decir, Kant cree que su propia noción de idea ayuda a comprender mejor el

19 «Pues nos las habemos aquí con una ilusión natural e inevitable (mit einer natürlichen und unvermeidlichen Ilusion) que se basa en principios subjetivos y los hace pasar por objetivos (...) Hay, por consiguiente, una dialéctica natural de la razón pura (...), [una dialéctica inherente a la razón humana de manera imposible de contrarrestar, y que aunque hayamos descubierto su maquinaria engañosa, no deja de exhibir sus falsas apariencias, ni de empujar a la razón incesantemente a extravíos momentáneos, que tienen que ser corregidos una y otra vez» (KrV A298/B354).

$20 \quad K r V$ A314/B370. 
asunto mismo al que se refieren las ideas platónicas, asunto que habría quedado oscurecido a causa de su confuso tratamiento por parte del filósofo griego.

El optimismo de Kant respecto de la validez de su doctrina contrasta sin embargo con la toma de cierta distancia a la hora de presentar su interpretación objetivista de las ideas en Platón. En este sentido, el pensador alemán advierte al menos en dos ocasiones acerca de las reservas hermenéuticas con las que debe acercarse el lector a la lectura de las ideas platónicas que él mismo presenta en la $K r V$ : en primer lugar, la consideración que se ha citado en el párrafo anterior se presenta a modo de contrapeso ante su propio reconocimiento de que no está interesado en desarrollar una investigación literaria para dilucidar el sentido que Platón daba a las formas. ${ }^{21}$ De hecho, y según se indica en estudios recientes sobre la recepción del pensamiento griego en Kant, todo apunta a que su aproximación a la filosofía platónica se dio a través de fuentes secundarias y, más en particular, a través de la Historia Critica de la Filosofía de Jakob Brucker, cuya interpretación se caracteriza por un fuerte sesgo matematizante de las ideas. ${ }^{22}$

En segundo lugar, en una nota al pie a sus consideraciones acerca de las ideas platónicas en la $\mathrm{KrV}$ reconoce de modo explícito que el tipo de lenguaje utilizado por Platón al hablar de ellas es muy susceptible de recibir una interpretación menos exagerada y más ajustada a la realidad de las cosas. ${ }^{23} \mathrm{~A}$ partir de estos dos puntos, resulta plausible sostener que el interés kantiano en las ideas platónicas es ante todo sistemático, es decir, aquello que interesa de ellas no es tanto su genuino papel en la filosofía platónica como su utilidad, tal y como son presentadas en la lectura objetivista tradicional, para ilustrar mediante su crítica un aspecto central de la comprensión kantiana de las estructuras que articulan el conocimiento objetivo.

Dentro del marco de la interpretación objetivista, como hemos visto en el epígrafe anterior, el error Platón que habría dado lugar a la teoría de las ideas consiste en la transposición de la validez subjetiva de lo ideal al plano de lo objetivo. La identificación de dicho error por parte de Kant se funda, como

$21 \operatorname{KrV}$ A313-4/B370. Según indica Kim $(2010,32)$, el conocimiento de Platón por parte de Kant, además de por la Historia Critica Philosophiae de Jakob Brucker, también por el Phädon de Moses Mendelssohn (cfr. Fistioc 2002 y Kim 2010, p. 32 n.68 y n.69 y p. 43).

22 Cfr. Kim 2010, cap. 1 y Fistioc 2002, pp. XX y 7ss..

23 «(...) En eso no puedo seguirlo, como tampoco en la deducción mística de esas ideas, ni en las exageraciones por las cuales él las hipostasió, por así decir; aunque el lenguaje elevado del que se sirvió en este campo puede muy bien recibir una interpretación más modesta y adecuada a la naturaleza de las cosas (...einer molderen und der natur der Dinge angemessenen Auslegung ganz wohl fähig ist)» (KrV A315/B372, nota al pie). 
también se ha visto, en la presuposición hermenéutica de que Platón concibe las ideas como objetos dados a una intuición de carácter intelectual. ${ }^{24}$

Los límites de esta lectura puede enunciarse de modo general como sigue. En primer lugar, y como ha sido puesto de manifiesto por distintos estudios acerca de la teoría platónica del conocimiento, ${ }^{25}$ el modelo de saber del que se sirve Platón en los diálogos como analogía para situar la discusión del acceso a las ideas no responde al de la captación intuitiva de objetos, ${ }^{26}$ sino al del saber técnico. En dicha forma de saber, el contenido ideal -es decir, lo irreductible en ella a lo dado empíricamente- no comparece de forma temática sino en la medida en que opera como criterio de orientación de lo que se hace en cuanto se hace. Dicho en terminología contemporánea, el conocimiento que versa sobre las ideas se acerca más a una forma de know-how que a una de know-that. Por ello, en los pocos pasajes de los diálogos en los que se tematiza directamente el tipo de contacto que se da entre el alma y la idea, el logro de tal conocimiento no se describe como la posesión de un objeto ideal en la conciencia, sino como un modo de disponerse el alma respecto de una instancia ideal en virtud de la cual obtiene la capacidad de orientarse con acierto en su vida (vid. esp. República 490a-b y Banquete 212a).

Como se acaba de decir, en los diálogos la idea no tiene propiamente la naturaleza de un contenido, sino la de una mediación ideal que permite orientarse adecuadamente en los distintos modos de tratar con uno mismo y con el mundo. Por otra parte, el conocimiento constatativo articulado discursivamente aparece siempre en los textos como un acceso derivado, secundario y de carácter propedéutico en relación con las ideas. Sirve para señalar y reflexionar acerca del modo en que uno está instalado en el mundo, pero no es el conocimiento que hace posible de forma originaria dicha instalación, esto es, no se identifica con la sabiduría. Como resulta especialmente claro en los diálogos tempranos, el propio manejo del logos, la capacidad de dialogar, depende también del tipo de disposición en el que el alma se encuentre respecto de la verdad de las cosas.

Sócrates en la República al hablar de las ideas, del mismo modo que Sócrates-Diotima en el Banquete al hablar de la idea de lo bello, ofrece un discurso acerca de un ámbito que, no obstante, no se hace presente de forma originaria en el mismo discurso. Mediante el logos se señala la idea como el único ámbito que hace posible el conocimiento $-\mathrm{y}$ se reduce al absurdo a quien trata de pensar y actuar al margen de lo ideal-, sin confundir por ello que el conocimiento que proporciona el propio discurso responde a un camino

24 Cfr. $K r V$ A853-4/B881-2.

25 Vid. p.ej. Nehamas (1992), Roochnik (1996), Woodruff (1999), Nussbaum (2001), Annas (2001), van Ackeren (2003).

26 Cfr. Trabattoni 2010. 
de naturaleza distinta al que se está recorriendo en la elaboración del propio discurso. En estos pasajes, se habla del acceso a lo ideal como un acceso que se da en la medida en que la idea resulta ser siempre el punto de orientación al que el sujeto, en su experiencia del mundo, está ya abierto de antemano, si bien de forma confusa. La filosofía en los diálogos de Platón-ya desde la Apologíano consiste en la obtención de contenidos, sino en el reconocimiento de los propios límites y, por tanto, de la propia situación respecto del genuino acceso a la realidad de las cosas en sus distintos modos. En esta medida, la filosofía se presenta como un camino de recuerdo de lo que ya se sabe, es decir, como una reorientación permanente del alma hacia la verdad de las cosas y de sí misma.

\section{IDEAS SIN TEORÍA DE LAS IDEAS (WolfGang Wieland)}

La interpretación no-objetivista de las formas platónicas encuentra uno de sus desarrollos más notables en la obra de Wolfgang Wieland Platon und die Formen des Wissens (Platón y las formas del conocimiento, 1982) (en adelante Platon). Esta monografía aborda el estudio de las ideas en Platón a partir de la atención rigurosa a las fuentes primarias y, al mismo tiempo, desde un profundo conocimiento de las estructuras del conocimiento humano tematizadas por la crítica kantiana así como de su posterior desarrollo en el neokantismo de Natorp y en la hermenéutica filosófica de corte heideggeriano. Como trataré de mostrar a continuación, la lectura propuesta por Wieland permite, en virtud precisamente de su conexión con la tradición crítica inaugurada por Kant, un mejor acercamiento hermenéutico a las ideas en Platón. ${ }^{27}$

La tesis principal de Wieland en Platon es que-a diferencia de lo que presupone de entrada la lectura objetivista tradicional- la noción platónica de idea no se identifica con el objeto de un conocimiento temático ${ }^{28}$ sino que consiste en la condición de posibilidad de todo conocimiento, en el punto de referencia (Bezugspunkt, Orientierungspunkt) ${ }^{29}$ bajo el que se orienta toda forma de trato con el mundo. La idea como tal puede a su vez ser tematizada en la teoría, pero resulta erróneo igualar el acceso a las ideas del con la consideración teórica de ellas. Al contrario, el modo originario de acceder a la idea es, como se decía, esencialmente atemático y se da justamente cuando esta opera como criterio de los modos de acceso al mundo y solo en la medida en que opera como tal. ${ }^{30}$ Al tratarla de modo temático, la idea aparece como objeto y pierde su estatuto originario de idea, esto es, criterio de orientación del trato con las cosas. Esto no invalida de modo absoluto el discurso sobre la idea, tan solo pone sobre

27 Vid. p.ej. Wieland 1999, p. 185.

28 Cfr. Wieland 1999, p. 103.

29 Idem, p. 101 y 148.

30 Cfr. idem, p. 148. 
aviso para no caer en la confusión de creer que mentar la idea en el discurso se identifica con el modo originario de conocerla.

Dicho conocimiento originario de la idea, tal y como se muestra repetidamente en los diálogos, resulta incomunicable de forma directa; Sócrates puede encaminar o encauzar a sus interlocutores hacia el conocimiento de la idea, pero no lo puede transmitir al modo de un contenido ni puede producirlo técnicamente en otros. En contra de lo que en ocasiones se interpreta, con ello Platón no está denunciando la el desconocimiento o incapacidad de su maestro respecto de según qué tipo de contenidos o discursos, sino que está apuntando más bien a los límites de comunicabilidad inherentes a la comprensión de la idea.

Para comprender correctamente lo anterior, resulta imprescindible tener en cuenta que Wieland considera que la naturaleza de las ideas en Platón debe comprenderse a la luz de los pasajes de Crátilo y República en los que se las presenta en el contexto del uso de artefactos, es decir, dentro de las estructuras que aparecen en las formas técnicas de saber. En ambos diálogos, la idea de un artefacto es aquello a lo que permanece orientado aquel que lo usa del modo adecuado a la condición de dicho artefacto. ${ }^{31}$ Ya sea quien lo produzca o quien lo use, el que tiene acceso a la idea es aquel que en su acción se orienta no a partir de la forma externa del artefacto, sino a partir de su función (Funktion). ${ }^{32}$ Tal conocimiento solo puede proporcionarlo realmente el conocimiento de uso (Gebrauchswissen), ${ }^{33}$ que no consiste en un conocimiento dirigido a un objeto, ${ }^{34}$ sino en un saber de orientación. En él, la idea cumple su función en la medida en que no comparece como tema, sino como criterio presente de forma indirecta en el obrar del agente. ${ }^{35}$ Por usar el ejemplo de Platón en República, la idea de las riendas para montar a caballo no es el conocimiento que tiene de ellas el pintor al representar los rasgos externos de las mismas, sino el conocimiento que tiene el jinete, esto es, el conocimiento que se hace actual y efectivo solo en el uso de ellas por parte del que sabe usarlas. Según señala Wieland, este es el modelo de comprensión conforme al cual Platón establece las ideas como paradigmas o ejemplares de las cosas. ${ }^{36}$

31 «Die Idee ist dasjenige, an dem der Gebrauchende orientiert bleibt, wenn er mit einer Sache bestimmungsgemäß umgeht» (Wieland 1999, p. 147).

32 «Diese Idee erfaßt man aber nicht schon dann, wenn man sich die äußere Form dieses Werkzeuges vorstellt. Man erfaßt sie erst dann, wenn man sich han seiner Funktion orientiert» (Wieland 1999, p. 146). En nota al pie, Wieland remite aquí a Crátilo 389a.

33 Idem, p. 146. Este tipo de conocimiento es el que, según este autor, Platón atribuye al demiurgo en el Timeo (cfr. Wieland 1999, p. 148).

34 Idem, p. 147.

35 «Es übt seine Funktion vollkommener Weise aus, wenn es sich zur Idee als seinem Orientierungspunkt nicht wie zu einem Objekt verhält» (ibid.). Cfr. idem, p. 148.

36 Cfr. ibidem. 
Por su parte, como ya se ha apuntado más arriba, el estatuto correspondiente a la consideración discursivo-temática de la idea es siempre y solo el de la reflexión -es decir, un acceso secundario-sobre las estructuras en las que la idea comparece de forma originaria. ${ }^{37}$ Los elementos que operan en el saber de uso, en efecto, pueden ser mentados por una teoría, pero el acceso al mundo que en el saber de uso acontece no puede ser revivido ni transmitido como tal por medio de una teoría. ${ }^{38}$ En efecto, puede hablarse del modo en que el jinete conoce las riendas, o el flautista la flauta, pero ninguno de los contenidos transmitidos en esta tematización directa puede sustituir el conocimiento efectivo que el jinete encarna al cabalgar o el flautista al tocar la flauta de modo competente. Como resulta claro, saber-constatar-que saben no equivale a saber lo que saben del modo en que lo saben. El modo originario de aparecer de las ideas no es el de un objeto, sino el del referente ejemplar al que siempre ya de entrada está referido un agente en su trato con las cosas en la medida en que trata con ellas $; 39$ su acceso a la idea será mayor cuanto más competente sea su trato con la cosa en cuestión. En este sentido, la introducción de las ideas en Platón no obedece a una duplicación de mundos de objetos -por un lado el mundo de los objetos sensibles y por el otro el de los suprasensibles-, sino a la explicitación de los distintos niveles que comparecen en el modo en que el sujeto está abierto a la realidad.

En el contexto de esta interpretación de la naturaleza ideal de las formas platónicas, la idea del bien ocupa naturalmente un lugar central. ${ }^{40}$ Como en el caso de las demás ideas, la comprensión del estatuto de la idea del bien en el pensamiento platónico no debe perseguir la infructuosa tarea de buscar una definición-explícita o implícita- de la misma en las fuentes platónicas. Puesto que las ideas comparecen originariamente solo en la medida en que funcionan como criterios de las formas de acceso al mundo, su estudio debe centrarse en el tipo de funciones que se les atribuyen en cada caso. En la República, de

37 «Eine recht verstandende Ideenlehre hat deswegen immer nur den Status einer Reflexionstheorie» (idem, p. 103). Leyendo a Wieland, Kim explica esto punto en los siguientes términos: «The Ideenlehre represents a theoretical distancing from the 'natural attitude', in which the ideai are actually operative» (Kim 2010, p. 191).

38 Cfr. Wieland 1999, p. 148.

39 Cfr. idem, p. 150. «...the inexplicit, yet always already understood points of reference of use-knwoledge» (Kim 2010, p. 291).

40 Cfr. Wieland 1999,p. 160. En su apéndice sobre la interpretación de Platón en Wieland, Kim parece pasar por alto la relevancia que la idea del bien juega en la lectura de este discípulo de Gadamer. Le dedica únicamente una mención tangencial en una nota al pie (vid. Kim 2010, p. 291 n.13). 
hecho, Sócrates rehúye explícitamente un tratamiento directo de la idea del bien y ofrece en su lugar un discurso acerca de las funciones que cumple esta idea. ${ }^{41}$

En esta línea, la primera función que Platón asigna a la idea de bien es la de posibilitar la plena realización de la justicia, ${ }^{42}$ esto es, su realización efectiva en el caso concreto (Einzelfall). ${ }^{43}$ De este modo, la prestación propia que caracteriza a la idea del bien es la adecuación del pensamiento o de la acción al caso particular. ${ }^{44}$ En este sentido, afirma Wieland, la idea de bien puede ser interpretada en términos kantianos como un principio de la facultad de juzgar (Urteilskraft). ${ }^{45}$ Siempre que el sujeto conoce o actúa, lo hace bajo la luz de un idea que le permite reconocer aquello en lo que se ocupa y disponerse hacia ello del modo adecuado en cada caso. Es el saber adecuarse a la cosa, el dar con la medida que, a su vez, no depende de una medida ulterior y es, por tanto, primaria dentro del ámbito de lo ideal. Tanto más competente será un sujeto cuanto mejor se adecúe su trato a la cosa, esto es, cuanto mayor sea la claridad ideal bajo la que se oriente en cada caso.

A la idea de bien se le asigna, además, otra función: la de fundar la utilidad o provecho (nutzstiftende Funktion) ${ }^{46}$ que potencialmente pueden proporcionar todas las demás cosas, incluidas aquellas formas de actuar que habitualmente son consideradas justas y virtuosas; ${ }^{47}$ sin el bien, nada puede resultar beneficioso. La idea del bien es comprendida aquí como el criterio que ante algo supuestamente bueno permite discernir si realmente lo es o no. ${ }^{48}$ Tal criterio opera siempre ya en alguna medida en toda acción, en la medida en que todo es hecho en función del beneficio que se supone que aporta. La competencia en dicho ámbito consiste, justamente, en la capacidad de evitar el autoengaño respecto a lo más importante, a saber, qué sea lo realmente bueno para uno.

De este modo, la subordinación de la comprensión de la utilidad o provecho de algo al acceso al bien por parte del sujeto hace visible el carácter estructuralmente teleológico de la acción humana y su particular vinculación con la autocomprensión; es decir, muestra que toda acción, en la medida en que es la realización de algo, se orienta ya siempre en función o a la vista de

41 Cfr. Wieland 1999, p. 161.

42 Cfr. 503e y ss.; Wieland 1999,p. 162. «Es handelt sich um einen Lehrgehalten, zu dessen Funktionen es gehört, dem Mangel an genauigkeit abzuhelfen, der mit der bislang erarbeiteten Bestimmung der Gerechtigkeit verbunden war» (idem, p. 165).

43 Cfr. idem, pp. 162-3.

44 «...dem Enizelfall gerecht zu werden» (idem, p. 163).

45 Cfr. idem, p. 164.

46 Idem, p. 167.

47 Cfr. idem, p. 165. Cfr. República 505a.

48 Cfr. idem, p. 166. 
una determinación de su fin último, de una representación del bien. ${ }^{49}$ Naturalmente, dicha representación del bien puede ser tematizada y explicitada mediante la reflexión, pero no en la acción misma, que es donde comparece de forma originaria.

Dada su naturaleza fundamenta en cuanto criterio valorativo último, la comprensión de la idea del bien permite ordenarlo todo en función de aquello que el hombre quiere realmente y hacia lo cual su pensamiento y acción se encuentran dirigidos de modo fundamental. ${ }^{50}$ En la medida en que el hombre actúa, su acceso al mundo está circunscrito dentro de la referencia al bien, es decir, se encuentra inmerso en un orden teleológico que le es dado en la acción y que no está a su disposición, puesto que constituye su condición misma de posibilidad. ${ }^{51}$ Esto significa que toda acción -como luego formuló la filosofía medieval- solo puede ser querida y efectivamente realizada sub ratione boni, esto es, en la medida en que el agente la toma como buena, es decir, como adecuada a lo que realmente desea y, por tanto, como un ejercicio -acertado o no- de autocomprensión.

Como se explica en República, la correcta orientación o disposición de la vida hacia la idea del bien se logra mediante un largo camino educativo en el que el aprendiz debe familiarizarse con los distintos modos de acceso al mundo y ejercitarse en ellos a través de experiencias concretas. Esta educación posibilita el acceso al bien, que se identifica con la capacidad de advertir aquello que corresponde al caso concreto -como se apuntaba antes-, es decir, de saber cuándo y cómo debe ser tomado un curso de acción, cuándo y cómo una acción resultará realmente buena. La comprensión del bien se identifica con la competencia plena en este ámbito.

A partir de lo anterior se advierte que la capacidad de acertar en dicha relación entre el caso concreto y el bien no se corresponde con un conocimiento temático, sino con un saber de uso orientado bajo la idea del bien. ${ }^{52}$ En efecto, la función que se asigna en República al conocimiento del bien no es la de captar un objeto, sino la de motivar y regular la actividad humana. ${ }^{53}$

Como señala Wieland, si esta interpretación de la idea del bien es correcta, con esta forma ideal de carácter supremo Platón está destacando un aspecto que

49 «Deshalb vermag der Begriff des Nützlichen bei Platon auch jene teleologische Ordnung des Handelns zu akzentuieren, die ihrer Natur nach immer an der Vorstellung eines Guten als einer Bestimmung ihres letzten Ziels orientiert bleibt» (idem, p. 167).

50 Cfr. idem pp.172-3 y 182.

51 «In diesem Falle fügt man Primärziele oder Teilzeile des Handelns in eine umfassendere teleologische Ordnung ein, die dem Handeln vorgegeben ist und nicht mehr zu seiner Disposition steht» (idem, p. 173).

52 Cfr. ibidem y p. 180.

53 Cfr. ibidem. 
pertenece a toda idea en la medida en que constituye un extremo normativo $\mathrm{y}$, por tanto, un punto de referencia respecto del cual el hombre se orienta en su trato con el mundo. ${ }^{54}$ Con ello se pone de relieve y se justifica el hecho de que ninguna virtud -como se muestra repetidamente en los diálogos tempranos- puede ser comprendida adecuadamente por separado, pues la posesión de cualquier excelencia, en la medida en que constituye realmente una excelencia, está como tal esencialmente referida al bien. ${ }^{55}$ Una idea, considerada en sí misma es propiamente un aspecto del bien (eine spezielle Gestalt des Guten $)^{56}$ y, por ello, solo en la medida en que es reconocida como tal resulta propiamente conocida.

La idea del bien aparece de este modo como el punto más alto de orientación (der oberste Orientierungspunkt) -el horizonte último de orientación-que comparece en toda acción como el orden teleológico que la posibilita. Por tanto, y como se ha dicho ya, dicho punto no se presenta originariamente en la forma de un objeto intendido. ${ }^{57}$ En la medida en que es el principio regulativo supremo del uso, es aquello que es comprendido cuando el trato con la cosa se ajusta a lo que esta es. Dicho trato como tal no puede ser anticipado en la forma de un conocimiento teórico ${ }^{58}$ en el sentido de que, como se ha dicho ya antes al hablar del tipo de conocimiento que corresponde al acceso a las ideas, saber de algo es propiamente saber tratar con ello. ${ }^{59} \mathrm{El}$ agente permanece orientado hacia el bien y hacia el cumplimiento de aquello que realmente quiere como agente en la medida en que en el trato con las cosas se adecúa a lo que estas realmente son. ${ }^{60}$ Por ello, el conocimiento de la idea del bien cumple según Wieland la función del saber de uso más alto (...eines obersten Gebrauchswissen). ${ }^{61}$

Ahora bien, el mismo autor resalta también el hecho que esta presencia regulativa del bien resulta operativa para Platón no solo en el ámbito de la práctica, sino que se extiende también al de la predicación, en la medida en que ésta consiste en la identificación de un caso concreto (Einzelfall) a la luz de una forma ideal (idealen Gebilde) ${ }^{62}$ y aparece por tanto como un tipo de práctica. Tal identificación o aplicación (Anwendung) revela la presencia de un momento normativo (ein normatives Moment), ya que requiere de un principio que la regule y conforme al cual reconocer lo particular como un caso de la

54 Cfr. idem, p. 175.

55 Cfr. idem, p. 182.

56 Ibidem.

57 Cfr. idem, pp. 176 y 180.

58 Cfr. ibid.

59 Cfr. idem, pp. 176-7.

60 Cfr. idem, pp. 179-80.

61 Idem, p. 180.

62 Cfr. idem, p. 183. 
norma. La idea del bien orienta también, en este sentido, el acceso predicativo al mundo. Por eso dicha idea es para Platón no solo principio de la acción, sino también principio del ser, es decir, de la determinación, y del conocer. ${ }^{63}$

Finalmente, la consideración de estas funciones que Platón atribuye al bien permite a Wieland también explicar el origen la posibilidad de tomar las ideas como objetos. Esta concepción de las ideas surge de una forma de tratar con las ideas que el mismo Platón tematiza en la República y que el intérprete alemán caracteriza como el uso matemático de las ideas (der mathematische Gebrauch).$^{64}$ Este uso, con el que Platón ejemplifica el acceso dianoético al mundo en contraste con el acceso dialéctico, es el único que trata las ideas como objetos. ${ }^{65}$ Se trata de un modo de pensar que toma las ideas como principios normativos absolutos, en el sentido de que se refiere a ellas como a un ámbito de validez determinado y autosuficiente y, en esta medida, separado de todo lo demás y por tanto externo al mundo. ${ }^{66}$

Las ideas, que originariamente comparecen como hipótesis, es decir, como presupuestos de los distintos modos por medio de los cuales se nos aparece el mundo, no son tratadas en el uso matemático como tales presupuestos, sino como principios. Dicho uso impide por su propia naturaleza tomar lo ideal como un caso de lo bueno y, por tanto, ascender desde la hipótesis hasta el bien como principio. La misma naturaleza de esta forma de pensar excluye la referencia al bien real para operar, ${ }^{67}$ pues la dianoia no puede acceder al principio anhipotético que es el bien porque ella misma trata las ideas como puntos de partida anhipotéticos y, por tanto, no como modos o aspectos del bien. Tomar la dianoia como el modo originario de conocer la realidad se identifica por tanto con la confusión de lo hipotético con lo anhipotético. De este modo, es en la consideración abierta por la dianoia donde las ideas adquieren por primera y única vez su estatuto objetual. En consecuencia, aquel que no está instruido en la dialéctica y cree que el pensamiento dianoético -objetivante- es el modo fundamental de conocer las cosas es el único que realmente cree que las ideas son objetos; únicamente él puede ser calificado de «platónico» en el sentido más tradicional -y por lo dicho hasta aquí erróneo- del término. ${ }^{68}$

El acceso a la idea del bien, en cambio, devuelve siempre al alma a la realidad de las cosas, en la medida en que es el punto de referencia que orienta todo uso, también el uso matemático de las ideas considerado como tal. El bien

63 Cfr. idem, p. 183 n.66. Cfr. República 517b y 508a y ss..

64 Idem, p. 183.

65 Cfr. ibidem.

66 Cfr. idem, p. 185.

67 Cfr. idem, p. 183.

68 «Daher ist der Mathematiker - und nur er- der 'Platoniker' im landläufigen Sinne des Wortes» (idem, p. 183). 
es aquello que comprende quien comprende qué estatuto real corresponde a cada forma de conocimiento $y$, por tanto, el que no toma el uso matemático de las ideas como un uso fundado en sí mismo, sino como una forma de conocimiento referida a un horizonte de orientación más allá de sí mismo. Por eso, en la alegoría de la caverna es la luz del sol, la luz auténtica, la que posibilita el retorno a ella, esto es, el modo auténtico de acceder al mundo de las sombras. La comprensión del bien-la noesis, que acontece únicamente en el pensamiento dialéctico- es la adecuada comprensión del mundo en su darse originario, en la medida en que regula la adecuación entre la formas de acceso -lo ideal en términos platónicos-y lo particular.

La idea del bien, por tanto, se encuentra más allá del ser (epekeina tes ousias), ${ }^{69}$ es decir, es inobjetiva (Ungegenständlich). A ella no le corresponde ningún objeto de la experiencia del mundo y, sin embargo, está esencialmente referida a dicha experiencia como su misma posibilidad. Su comprensión, por tanto, se corresponde con la capacidad del hombre para orientar de modo adecuado a la verdad de las cosas -justo- los distintos modos mediante los que se desenvuelve en el mundo. ${ }^{70}$

\section{REFERENCIAS BIBLIOGRÁFICAS:}

ANNAS, J. 2001: «Moral Knowledge as Practical Knowledge.» Social Philosophy and Policy, 18 (2), 236.

FISTIOC, M.C. 2002: The Beautiful Shape oft he Good: Platonic and Pythagorean Themes in Kant's Critique oft he Power of Judgement. New York \& London: Routledge.

KANT, I, Crítica de la razón pura, tr. M. Caimi. México D.F.: Fondo de Cultura Económica, , 2011.

KIM, A. 2010: Plato in Germany. Kant-Natorp-Heidegger. Sankt Augustin: Academia Verlag.

NEHAMAS, A. 1992: «What Did Socrates Teach and to Whom Did He Teach It?», The Review of Metaphysics, 46 (2), 279-306.

NUSSBAUM, M. C. 2001: The fragility of goodness: luck and ethics in Greek tragedy and philosophy. Cambridge: Cambridge University Press.

ROOCHNIK, D. 1996: Of Art and Wisdom Plato's Understanding of Techne. Penn State University Press.

TRABATTONI, F. 2010: «Il sapere del Filosofo» en VEGETTI, M. 2010: Platone. La

69 República 509b.

70 «...sie verliet ihrem Inhaber die Fähigkeit, dieser Welt und sich selbst in ihr Gerecht zu werden» (idem, p. 185). 
Repubblica, vol. V, Napoli: Bibliopolis, pp. 151-186.

VAN ACKEREN, M. 2003: Das Wissen vom Guten: Bedeutung und Kontinuität des Tugendwissens in den Dialogen Platons. Amsterdam: Grüner.

WOODRUFF, P. 1990: «Plato's Early Theory of Knowledge», en S. EVERSON (ed.), Epistemology. New York: Cambridge University Press, pp. 60-84.

WIELAND, W. 1999: Platon und die Formen des Wissens. Göttingen: Vandenhoeck und Ruprecht.

Miquel Solans Blasco es doctor en filosofía

Lineas de investigación

Platón, filosofía antigua, ética y teoría de la acción, hermenéutica.

Últimas publicaciones

«Libertad humana y libertad divina. Una lectura de Cultura y verdad a la luz de la imaginación trascendental» en Naturaleza y libertad. Revista de estudios interdisciplinares, 9-2017.

Email:msolans@alumni.unav.es 